\title{
La política educativa y su compromiso con la equidad social y el desarrollo sostenible
}

\author{
Nancy Torres Victoria ${ }^{1}$ \\ Centro de Investigación y Docencia en Educación (CIDE), Universidad Nacional \\ Heredia, Costa Rica
}

Recibido 27 de octubre de 2009 • Aceptado 02 de diciembre de 2009

Resumen. Este artículo se centra en el análisis de dos aspectos de la política educativa vigente en Costa Rica: las declaraciones que contiene el documento de política educativa sobre su compromiso con la equidad social y el desarrollo sostenible, tema que tiene estrecha relación con la educación rural, su conceptualización, su planificación, su desarrollo. Asimismo, traza un breve recorrido por el desarrollo del derecho a la educación en la normativa nacional, y muestra los avances en materia de cobertura a los grupos vulnerables del país, con la promulgación de nuevas leyes que estos sectores han venido conquistado a partir de luchas y demandas organizadas.

Palabras clave. Educación rural, políticas educativas y educación rural, derecho a la educación, desarrollo sostenible.

Abstract. The article centers its analysis in two areas of the current educational policies in Costa Rica: the statements that the document contains about its commitment with the social equity and the sustainable development, subject that has a strong relationship with rural education, its conceptualization, its planning, and its development. At the same time, it traces a brief description of the development of the right to education in the nation's politics, showing advances of the country's vulnerable groups, with the promulgation of new laws that these sectors have been reaching through fights and organized demands.

Key words. Rural education, educational politicies and rural education, right to education, sustainable development.

\footnotetext{
${ }^{1}$ Historiadora, Máster en Planificación Curricular de la Universidad de Costa Rica (UCR). Profesora y miembra del equipo de investigación y extensión de la División de Educación Rural del Centro de Investigación y Docencia en Educación (CIDE) de la Universidad Nacional, Costa Rica. Correo electrónico: ntorres65@gmail.com
}

La Revista Electrónic@ Educare está indizada en el Catálogo LATINDEX.

Artículo protegido por la licencia Creative Commons. 


\section{Introducción}

En este artículo se pretende analizar, a la luz de la política educativa vigente, los compromisos que declaran la intención de cerrar brechas entre lo rural y lo urbano y de atender, específicamente, con programas especiales las diferencias existentes en lo rural disperso.

Actualmente, nadie pone en duda el importante rol que juega la educación en la vida colectiva e individual de la sociedad. Una persona que tenga un nivel alto de educación, no solamente está en capacidad de acceder a mejores empleos, sino que podría utilizar, de forma más racional, los recursos a su alcance, hacer un mejor uso de la información, tener mejores opciones para resolver los problemas que se le presentan; pero, sobre todo, entender mejor su cultura respetando y valorando la riqueza de la diversidad. La educación es, entonces, la vía indiscutible para lograr mejores condiciones de vida para todos los que habitamos en el planeta, mediante el desarrollo de mecanismos sociales de inclusión a la vida política, mayor equidad en las oportunidades, respeto por la divergencia y las libertades civiles; en síntesis, se propende por una educación que genere una ética para el desarrollo sustentable, por medio de la intensificación del conocimiento y la conciencia de lo público.

\section{Derechos educativos}

Lo que se conoce como el marco de referencia de todos los programas y planes educativos es, hasta ahora, la Política Educativa hacia el siglo XXI, presentada por el Ministro de Educación Dr. Eduardo Doryan, al presidente José María Figueres, en 1994. En este documento, se recogen los principales acuerdos y orientaciones de la Conferencia Mundial de Educación para todos, realizada en Jontiem, en 1990. Este marco referencial propone la política educativa de estado, para el período comprendido entre 1995 y 2005. A pesar de que su vigencia se preveía para diez años, ya lleva vigente trece años; después de tres administraciones ejercidas por dos partidos políticos. Sin embargo, el consenso en torno a la Política Educativa hacia el siglo XXI, rápidamente, se ha vuelto frágil. Como bien lo reseña Meoño (2007); en su informe sobre el Estado del Derecho a la Educación

En 1995, se presentó a la Asamblea Legislativa para su aprobación del proyecto EDU-2005, que fue rechazado y que, de haber sido aprobado, le hubiera dado a dicha política educativa el rango de ley de la República. Este proyecto se elabora con la idea de profundizar las políticas educativas contenidas en la Política Educativa hacia el Siglo XXI, y pretendía ampliar los alcances y convertir dichas políticas en ley de la República, lo que hubiera sido muy afortunado para el país. (p. 22)

Por ejemplo, proponía dos años de enseñanza preescolar gratuita y obligatoria, la reducción del número de estudiantes por aula, la enseñanza del idioma extranjero y de la informática desde el nivel de preescolar, incentivos económicos y profesionales para docentes, la universalización de la educación secundaria, el acceso efectivo para las personas con necesidades educativas especiales, y la erradicación total del analfabetismo, entre otras disposiciones.

Igualmente, sigue vigente la Ley Fundamental de Educación de 1957, que, ha sido complementada con programas y proyectos, tal como lo afirma el I Informe del Estado de la Educación del 2005:

las diferentes administraciones se han dado a la tarea de desarrollar en sus respectivas administraciones programas que remozan determinados aspectos de la educación, por ejemplo en el período de 1990-1994 el esfuerzo se centró en la política curricular y en la 
educación de adultos, la administración siguiente generó diagnósticos y declaraciones sobre la obligatoriedad de la educación preescolar, se aumentó el número de días lectivos de 169 a 200, y la reforma constitucional que garantiza no menos del 6\% del PIB para la educación; se impulsaron políticas específicas sobre la enseñanza de los idiomas, la informática y la introducción de la Telesecundaria, así como la elaboración de nuevos textos didácticos y el establecimiento de las pruebas de finalización del tercer ciclo. (p. 53)

En consecuencia, los pilares jurídicos de la educación costarricense son: la Constitución Política, la Ley General de Educación, las orientaciones del Consejo Superior de Educación y la Política Educativa Hacia el Siglo XXI. Esta última es el documento que, con mayor claridad, responde a los retos que la realidad actual plantea.

\section{Brechas que limitan el desarrollo del país}

Es necesario detenerse en los apartados del documento de la Política Educativa hacia el Siglo XXI, que se refieren al tema de la brecha entre lo rural y lo urbano, a la prioridad que debe brindarse en la atención de las zonas rurales, y en lo referente al derecho a la educación. Así, en este documento, en el apartado que contiene las bases conceptuales de las políticas educativas hacia el siglo XXI, se reconoce la existencia en Costa Rica de cuatro tipos de brechas que impiden o limitan el desarrollo sostenible del país. En este punto es importante detenerse, porque tiene relación con la brecha entre lo rural y lo urbano que es un tema clave por analizar. Las cuatro tipos de brechas reconocidas son: la brecha conceptual, la brecha del conocimiento, la brecha social y la brecha competitiva.

Brecha conceptual. Se identifican dos concepciones distintas de desarrollo propuestas desde el Estado: "Hay que cerrar la brecha conceptual entre economía y ecología y orientar las políticas públicas para que Estado y el mercado se muevan en la dirección de la administración sustentable de los recursos" (Chavarría y Tovar, 1998, p. 6). La conceptualización de lo económico es mucho más rica, desarrollada y planteada de manera positiva que la que existe en la conceptualización de lo ecológico. De este modo, todos tenemos más claro hacia dónde vamos en lo relacionado con el tema económico, y qué es lo que habría que hacer, pero no tenemos tan claro el panorama en lo relacionado con el uso de nuestros recursos. Habría que orientar las políticas públicas y educativas para que desarrollen más y mejores posiciones conceptuales en relación con el manejo, el uso, el disfrute, la conservación y la recuperación de los recursos naturales del Estado.

La función que tiene que cumplir la educación, para reconceptualizar lo ecológico con la misma fuerza y claridad con la que se conceptualiza lo económico en el país, es propiciar que el currículo que desarrolla la escuela rural parta de la realidad ecológica y responda a las preguntas que esa realidad tiene, que sean respuestas construidas con y desde lo rural, lo local, sin negar, ni obviar la visión económica o de mercado, pero dando igual valor a los aspectos relacionados con la calidad de vida. Por ejemplo, si hay un río en mi comunidad con condiciones excelentes para la producción de energía, yo puedo visualizarlo en función de que allí se pueda construir una gran represa, que, al final, generaría una cantidad considerable de dinero. Pero, igualmente, se debe trabajar inculcando actitudes responsables hacia su cuidado, de forma que, tanto en la actualidad como en el futuro, se tenga un río limpio, sano, refrescante, en función de lo que significa y aporta en términos de salud, agua potable y calidad de vida. Así, la escuela debe convertirse en un ente que promueve una cultura ambientalista que lleve al desarrollo sostenible, para que los miembros 
de una comunidad puedan decidir, en cada momento histórico, lo que es más conveniente, es decir, lo que es más rentable y relevante en términos de desarrollo humano.

Brecha del conocimiento. "Cerrar esta brecha es garantizar los conocimientos para detener el uso irresponsable de los recursos ambientales" (Chavarría y Tovar, 1998, p. 7). Se pretende, así, crear mayor conciencia de la existencia limitada de los recursos naturales. Hasta hace unos treinta años se educaba sin hacer mayor énfasis en este tipo de temas, pues no existía plena conciencia sobre la posibilidad de que algunos recursos, tales como el agua y el aire, llegarían a agotarse o a deteriorarse al punto de que la humanidad tuviera que prescindir de ellos. De ahí la importancia de mantener la alerta sobre este tema en las generaciones de relevo, las que, a la postre, sufrirán, con mayor rigor, las consecuencias del deterioro y escasez o ausencia de este tipo de recursos tan vitales en la naturaleza.

La brecha de conocimiento que existe entre las poblaciones rural y urbana no consiste en que la urbana tenga más conocimiento que la rural, sino que esta última no posee conocimiento de su verdadera realidad. No se trata, entonces, de que todos sepamos lo mismo, sino de que tengamos el suficiente conocimiento requerido para comprender nuestro mundo. Las poblaciones rurales adolecen del conocimiento universal y del conocimiento específico, los cuales les permitiría conocer su realidad. En esto consiste, realmente, la brecha, porque pareciera que se pretende que la población rural viva y actúe con sus recursos como se vive y se actúa desde la visión urbana. Por ejemplo, cuando se piensa que una población está muy subdesarrollada porque ni siquiera le llega la Coca-Cola, porque no tienen cable o porque no hay servicio de cajeros automáticos, posiblemente, no estamos pensando en los conocimientos requeridos para tener calidad de vida en el ámbito rural.

Brecha social. Para cerrar esta brecha "es necesario mejorar el acceso y la calidad de los servicios básicos a todos los sectores de la sociedad, pero, especialmente, a los más necesitados" (Chavarría y Tovar, 1998, p. 7). Se manifiesta de manera clara y precisa, en el principal documento político vigente, la necesidad y la intención de favorecer especialmente a los más necesitados con servicios básicos de calidad -y la educación es uno de los fundamentales-. Por tanto, en esta declaratoria se muestra la reconocida importancia que tiene la apertura de nuevas oportunidades de acceso a la educación para todos los habitantes del país. Todas las estadísticas y la simple observación de la realidad demuestran que, es en las zonas rurales, pero, principalmente, en la zona rural dispersa donde viven las personas con menos recursos y oportunidades, pues no existen reales posibilidades de acceso a la educación de calidad y con pertinencia en todos sus niveles.

Ésta es la brecha que, con mayor claridad, expone el problema de cara a los desafíos en la educación. Se trata de abrir oportunidades reales, para que toda la población tenga acceso a una educación que le permita acceder a otros niveles de la educación; pero no sólo aprender a leer y a escribir, sino a obtener un título de conclusión de la educación general básica que permita acceder a la educación técnica, universitaria o parauniversitaria. Y, en todo caso, para poder acceder a puestos de trabajo con una remuneración que propicie las condiciones para una vida digna y que permita comprender el mundo de manera integral; para poder construir, junto con otros, el porvenir de sus comunidades.

Al respecto, resulta ilustrativo el análisis que aparece en el X Informe del Estado de la Nación:

Durante el 2004, Costa Rica sufrió un deterioro en las oportunidades para que su población tenga una mejor calidad de vida, que se expresó en un aumento de la pobreza, una reducción en los ingresos laborales de todos los estratos y una disminución del ingreso social que el 
Estado proporciona a los hogares. Si bien, en términos generales, este deterioro afectó a la mayoría de los habitantes del país, los más golpeados fueron los sectores sociales más pobres y, por tanto, más vulnerables. Este magro desempeño ocurre en una nación que a lo largo de los últimos quince años experimentó progreso social, aunque sin lograr una conexión entre la inversión en capacidades humanas (salud y educación, por ejemplo) y el objetivo de convertirse en una sociedad más equitativa. (Programa Estado de la Nación en Desarrollo Humano Sostenible, 2004, p. 128)

Brecha competitiva. Ésta hace énfasis en la necesidad de cuidar la formación del capital humano, y destaca el indiscutible rol que tiene la educación para poder cerrar esta brecha.

Si bien en el documento se identifica, claramente, el rol de la educación como motor del desarrollo sustentable, se hace énfasis en la perspectiva de la educación como elemento dinamizador de la capacidad emprendedora, la creación de la cultura productiva y la capacitación de mano de obra calificada para la reproducción de la riqueza. Una educación de calidad debería tender a desarrollar capacidades imaginativas, creativas y recreativas de la población desde su realidad; pero, también, superar las condiciones de esta realidad. Por ejemplo, en una población rural marginal, se educará desde esta realidad, pero tendiendo a superar esta marginalidad; se debe tender a desarrollar las competencias que le permitan a las personas pensar, imaginar, construir, con otros y competir con estos otros. Entonces, la educación tiene que desarrollar competencias y sentido de competitividad, pero esta competitividad será sana en la medida en que se desarrolle a la par del sentido de la solidaridad y del trabajo colaborativo.

Como bien lo indica la Declaración de la campaña Latinoamericana por el derecho a la educación (2007), con ocasión de la reunión de los Ministros de Educación de Iberoamérica, en Valparaíso (Chile).

Recibir educación y educación de calidad es un derecho fundamental y una obligación asumida por los Estados como una de sus funciones fundamentales. La educación, además de ser eficaz y eficiente, debe respetar los derechos de todas las personas, ser relevante, pertinente y equitativa. Ejercer el derecho a la educación es esencial para desarrollar la personalidad e implementar los otros derechos humanos, por lo que a nadie se le puede excluir de ella. El derecho a la educación significa el derecho a aprender a lo largo de la vida y está fundado en los principios de obligatoriedad y gratuidad, y en el derecho a la no discriminación, como se verá más adelante. La educación que se recibe debe desarrollar las competencias necesarias para participar en los diferentes ámbitos de la vida humana y construir proyectos de vida con relación a los otros. (p. 2)

En Costa Rica, se reconocen las notorias diferencias existentes entre las poblaciones rural y urbana, se llama la atención sobre la inexistencia de un acuerdo nacional a largo plazo acerca del tema educativo. Entre las acciones que debería realizar el sector educativo para promover la sustentabilidad, se recomienda "orientar la educación hacia el desarrollo sostenible", describiendo una acción como la ampliación de la cobertura en el sector rural como una de las prioridades por lograr. En cuanto a los retos de la educación costarricense es importante destinar "instrumentos y recursos donde se encuentren los más débiles. Atendiendo especialmente la problemática de las escuelas unidocentes y..., de las personas que por una razón involuntaria han sido marginadas del proceso educativo convencional" (Chavarría y Tovar, 1998, p. 104). 


\section{Política educativa hacia el siglo XXI}

El detallado análisis del documento Política educativa hacia el siglo XXI, a la luz de los avances conceptuales en materia de derecho a la educación se hace relevante, porque es el texto en el cual se demarcan las orientaciones fundamentales para desarrollar los planes, los programas y las acciones educativas en el ámbito nacional. Asimismo, porque contiene las bases de la política educativa vigente desde 1998 y pretendió, desde su concepción, trascender los períodos gubernamentales para convertirse en una política de Estado.

Finalmente, desde los cinco desafíos que destaca la política vigente, se enfatiza sobre la urgencia de "destinar los instrumentos y recursos más fuertes donde están los más débiles". (Chavarría y Tovar, 1998, p. 104). De esta manera, se recalca la intención del Estado en su política educativa vigente, de cerrar brechas, de atender a las poblaciones que más lo requieren, con lo que se evidencia el interés y la necesidad de que la educación, como derecho humano, sea accesible a todos los rincones del país, es decir, es clara la intencionalidad, desde lo documental, lo declarativo y lo programático de compromiso del Estado por realizar esfuerzos para que la educación con calidad, equidad, eficiencia y dignidad sea una realidad para todos los habitantes del territorio costarricense.

Es importante, también, destacar la claridad existente en la política educativa en relación con las prioridades de los recursos: “donde están los más débiles”. En primer lugar, en el medio rural; en particular, en las escuelas multigrados (o unidocentes). En segundo lugar, en el medio urbano, la atención de las poblaciones marginales y en tercer lugar, en la atención de las personas con necesidades educativas especiales. Cabe hacer notar la invisibilización de la educación para los pueblos indígenas, en los criterios de priorización para la distribución de la inversión social en educación.

Más adelante, el documento Política educativa hacia el siglo XXI (Chavarría y Tovar, 1998) define sus objetivos por alcanzar. Entre ellos hay que destacar que, de nuevo, el primero que se propone expresa, claramente, el indiscutible compromiso con la educación rural: "Cerrar las brechas existentes entre la calidad de la educación que reciben los estudiantes de las áreas urbanas y rurales y eliminar la diferenciación entre las instituciones educativas de las áreas urbanas marginales y no marginales" (s. p.). Este objetivo reconoce las innegables diferencias en cuanto a la calidad y los servicios educativos existentes en las dos zonas.

El componente ambiental es un elemento clave que transversa toda la política. Es clara la estrecha relación que se puede establecer entre lo relativo al tema ambiental y la educación rural. Hacer explícita en la educación la relación entre población, recursos físicos y territorio, en el marco de la educación rural, es una necesidad, ya que en la zona rural se cuenta con mayor concentración de posibilidades y oportunidades de aportar para enriquecer y conservar lo ambiental, lo que implica una mayor responsabilidad con el planeta y con el país sobre este tema. La educación rural tiene que ver con todo eso, con educación para el cuidado de las aguas, con educación para las siembras propias, autóctonas y el cuidado de las tierras, la educación para la reflexión sobre la relación persona-naturaleza y con muchos temas relacionados con el ambiente. No por esto se debe depositar toda la responsabilidad de preservar y de conservar los recursos naturales en las personas que habitan en las zonas rurales; sí hay que tener claro que, por el hecho de vivir allí, el habitante rural tiene una responsabilidad local.

Todo esto tiene consecuencias políticas, ya que para hacerse cargo de esta responsabilidad en lo educativo, deben darse las condiciones desde el Estado. Para ello, se requiere garantizar políticas muy definidas, recursos, mecanismos y vías de regulación, de aplicación, es decir, hay que ir más allá de las declaraciones programáticas bien elaboradas, pues se requiere generar una conciencia y una ética en las que se sostengan las conductas de cuidado y uso moderado de los recursos; por 
otro lado, también, en el campo educativo, deben desarrollarse acciones para que, desde los ámbitos urbanos, se controlen, se regulen y se examinen los desórdenes y los desmanes que ocurren y que tienen impacto e incidencia directa en el ambiente rural.

Como ya se ha mencionado, el marco jurídico de la política educativa costarricense lo orientan: la Política Educativa Hacia el Siglo XXI, la Constitución Política, la Ley Fundamental de Educación y lo que dictamine el Consejo Superior de Educación. La política educativa que rige pretendió trascender los períodos gubernamentales. Se elaboró mediante un complejo proceso de consulta y de participación que duró alrededor de seis meses para, finalmente, ser aprobada como documento oficial el día 8 de noviembre de 1994, en la sesión número 8294 del Consejo Superior de Educación, en forma unánime y mediante acuerdo firme.

Dos principios de la política educativa vigente reconocen la educación como derecho fundamental de todas las personas que habitan en el territorio de Costa Rica. Hay que destacar que la única condición que se menciona, aquí, para ser acreedor del derecho es estar habitando el territorio costarricense. Es decir, se confiere el derecho por encima de las calidades o condiciones del estatus migratorio, de la edad, del estado civil, de las condiciones de salud, credo, raza, etc.

El Artículo VI, de las Normas Básicas Reguladoras del Proceso Educativo, en el cual se consignan los principios de la educación costarricense declara:

La orientación y satisfacción del derecho a la educación descansa en los principios constitucionales, el derecho internacional vigente en el país, en las disposiciones de la Ley Fundamenta de educación, número 1261 de Septiembre 25 de 1957 y en las determinaciones del Consejo Superior de Educación. (Chavarría y Tovar, 1998, p. 119)

El mismo documento, en el Artículo VII señala: "La satisfacción y el reconocimiento debido al derecho a la educación en las etapas y circunstancias pertinentes, requieren de la obligada participación de la familia y la comunidad organizada" (p. 119).

Y más adelante destaca y aclara, en el artículo IX: "El proceso formal regular se desarrollará en forma presencial en las tres instituciones educativas, que funcionarán como agencias del sistema, sin perjuicio de los procesos propios de la Educación Abierta y de la Educación a Distancia, cuando las circunstancias y las condiciones personales del educando así lo requieran”. En estos dos artículos, que tocan los principios educativos de la Política, se puede encontrar la médula conceptual en la cual se basa la propuesta de este trabajo. Se parte de las capacidades locales -aquí se incluyen, obviamente, la participación y el interés de las familias organizadas en las comunidades rurales- y a causa de las circunstancias especiales de ubicación y distanciamiento de las zonas rurales -lejos de los centros académicos y de los centros de desarrollo- que tiene sentido la propuesta de organizar y reunir los esfuerzos de las comunidades liderados por el docente rural, para abrir posibilidades y oportunidades educativas a las personas adolescentes que terminan el II ciclo, con el fin de que puedan continuar con sus estudios de secundaria en la modalidad de Educación Abierta.

\section{El derecho a la educación y su implementación en la normativa nacional}

La Ley Fundamental de Educación, de 1957, establece los fines generales para cada uno de los niveles educativos, la organización del sistema educativo y la rectoría del sector por parte del Consejo Superior de Educación. Igualmente, regula la formación docente, la educación privada y la educación no formal. 
El Código de la Niñez y la Adolescencia (Costa Rica. Asamblea Legislativa, Ley 7739), aprobado el 06 febrero de 1998, le da soporte legal al derecho a la educación para las personas menores de edad que habitan en el territorio costarricense, más allá del texto constitucional y de la Ley Fundamental de Educación. Los artículos del 56 al 72 de este código están consagrados, expresamente, al ejercicio del derecho a la educación. En materia de obligatoriedad y gratuidad del sistema educativo, el Código incluye disposiciones específicas y señala como responsable de su cumplimiento al Ministerio de Educación Pública (MEP) y a las personas mayores de edad: padres, madres o adultos encargados. Igualmente, en el Artículo 56 se explicita la obligación estatal de cara a la educación en y con derechos humanos.

Otro documento que hace referencia directa a los derechos humanos, en materia de educación, es el Convenio centroamericano sobre unificación básica de la educación (Coordinación Educativa y Cultural Centroamericana, 1966); en él se lee: "La educación debe... reforzar el respeto de los derechos humanos y de las libertades fundamentales" (ordinal 5, inciso 1.a).

Asimismo, el Artículo 28 de la Convención de los Derechos del Niño (Oficina del Alto Comisionado de las Naciones Unidas para los Derechos Humanos, 1989), contempla la responsabilidad de los estados firmantes a implantar la enseñanza primaria gratuita y obligatoria para todos sus habitantes; también se destaca el compromiso de los estados a fomentar la enseñanza secundaria y profesional.

\section{Apreciación final}

Como bien puede deducirse, es necesario reconocer que, en la Constitución Política y en la Ley Fundamental de Educación no se contempla el ejercicio del derecho a la educación por parte de los grupos y sectores más vulnerables de la población; en consecuencia, tal como lo afirma Meoño (2008, p 8): “estos grupos han venido conquistando sus propios derechos, su propia legislación”. Por supuesto, se reconoce que en el contexto histórico en el cual se creó la Ley Fundamental de Educación, no se había conquistado el reconocimiento social de los derechos de estos grupos.

Para ese momento, el tema del derecho a la educación como un derecho universal aún no se había posicionado como tal; lo mismo puede decirse de los derechos de la población con necesidades especiales.

El desarrollo de la legislación ha venido complementando con nuevas leyes los derechos de la población más vulnerable, como puede comprobarse, con la Ley de creación de la Defensoría de los Habitantes de la República, en 1992 (modificada en 1994); las personas con necesidades especiales, con la Ley de Igualdad de Oportunidades (7600), de 1998; los pueblos indígenas, con la Ley de Desarrollo Autónomo de los Pueblos Indígenas; o las mujeres, con la Ley de Violencia Doméstica, de 1996, la Ley del Instituto Nacional de las Mujeres, de 1998; y las madres adolescentes, con la Ley General de Protección a la Madre Adolescente, de 1997.

En otras palabras, lo que puede observarse al estudiar la nueva legislación complementaria en materia de educación, es que ésta se ha ido actualizando a medida que las exigencias nacionales e internacionales así lo han requerido.

Debe quedar claro que, en materia de mejoramiento de la calidad de vida y desarrollo sostenible en las comunidades rurales, y como respuesta a las particularidades y necesidades específicas de este tipo de poblaciones, la educación y el cumplimiento de este derecho es la respuesta que puede llegar a potenciar un mayor bienestar y equidad en las comunidades rurales. 


\section{Referencias bibliográficas}

Campaña Latinoamericana por el Derecho a la Educación (CLADE). (2007). Reunión de los Ministros de Educación de Iberoamérica en Valparaíso (Chile), realizada los días 26 y 27 de julio de 2007. Recuperado el 3 de mayo, 2010, de Ceaal.org/

Chavarría, S. \& Tovar, F. (1998). Política educativa hacia el siglo XXI: Sus bases conceptuales. San José, Costa Rica: Ministerio de Educación Pública.

Coordinación Educativa y Cultural Centroamericana. (CECC). (1966). Convenio centroamericano sobre unificación básica de la educación. (Segunda Reunión del Consejo Cultural y Educativo de la Organización de los Estados Centroamericanos, realizado en San Salvador). Recuperado el 22 de abril de 2010 de http://www.apse.or.cr/webapse/docum/docu01.htm

Costa Rica. Asamblea Legislativa. (1998). Código de la Niñez y la Adolescencia. (Ley de la República de Costa Rica No. 7739, del 6 de febrero de 1998). San José, Costa Rica: Autor.

Costa Rica. Ministerio de Educación pública. (1957). Ley Fundamental de Educación. 25 de septiembre de 1957. San José, Costa Rica: Asamblea Legislativa.

Meoño, R. (2008). Informe sobre el Derecho a la Educación. San José, Costa Rica: Manuscrito inédito.

Oficina del Alto Comisionado de las Naciones Unidas para los Derechos Humanos. (1989). Convención sobre los Derechos del Niño. Asamblea General de Naciones Unidas. 20 de Noviembre 1989. Resolución 44/25. Ratificado en Costa Rica por la Ley 7184 publicada en La Gaceta, No. 149 del 9 de Agosto de 1990, s. p.

Programa Estado de la Nación en Desarrollo Humano Sostenible. (2004). Décimo informe sobre el Estado de la Nación. San José, Costa Rica: Proyecto Estado de la Nación.

Programa Estado de la Nación en Desarrollo Humano Sostenible. (2005). CONARE (Coord.). I Informe sobre el Estado de la Educación Costarricense. San José, Costa Rica: Proyecto Estado de la Nación. 